\title{
Proceeding
}

Supplementary Issue: Spring Conferences of Sports Science. International Conference on Psychology of Education Sciences \& Lifestyle.

\section{Effect of physical therapy rehabilitation program (exercise-therapy) on Irritable Bowel Syndrome}

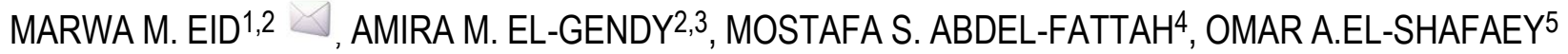 \\ ${ }^{1}$ Department of Physical Therapy, Faculty of Applied Medical Science, Taif University, Saudi Arabia \\ 2Department of Physical Therapy for Surgery, Faculty of Physical Therapy, Cairo University, Giza, Egypt \\ ${ }^{3}$ Department of Basic Sciences, Faculty of Physical Therapy, Cairo University, Giza, Egypt \\ ${ }^{4}$ Department of Physical Therapy for Cardiovascular, Respiratory Disorders and Geriatrics, Faculty of Physical \\ Therapy, Cairo University, Giza, Egypt \\ ${ }^{5}$ Cairo University ,Egypt
}

\begin{abstract}
To evaluate the effect of physical therapy rehabilitation program consisted of moderate intensity aerobic exercise training (60\% MHR) (Maximum Heart Rate) treadmill training on irritable bowel syndrome (IBS) symptom and quality of Life in post cholecystectomy patients. Thirty post cholecystectomy patients complaining from irritable bowel syndrome were recruited for this study; age ranged from 25 to 40 years, Patients were divided randomly into two equal groups, Group (A): received 4 weeks moderate intensity aerobic training program (60\% MHR treadmill training (3 sessions per week) plus routine medical treatment protocol, Group (B): received routine medical care only. Patients assessed by Irritable Bowel Syndrome Symptom Severity Score (IBS-SSS) and Irritable Bowel Syndrome Quality of Life (IBS-QOL), Assessment was carried out before treatment and 4 weeks after treatment in both groups. Results showed that there was a significant decrease in post treatment values of IBS-SSS and IBS-QOL in comparison to pre-treatment values at both groups with ( $p$-value < .001). Comparison between both groups post treatment showed significant difference in IBS-SSS and IBS-QOL variables, in group (A) more than group (B). Moderate intensity aerobic exercise training $(60 \% \mathrm{MHR})$ for 4 weeks is effective in treatment of IBS post cholecystectomy through reducing IBS symptoms and improving Quality of Life.

Keywords: Irritable Bowel Syndrome (IBS); Aerobic exercises; Cholecystectomy; Quality of life; Symptom severity score.

Cite this article as:

Eid, M.M., El-Gendy, A.M., Abdel-Fattah, M.S., \& El-Shafaey, O.A. (2020). Effect of physical therapy rehabilitation program (exercise-therapy) on Irritable Bowel Syndrome. Journal of Human Sport and Exercise, 15(3proc), S907-S913. doi:https://doi.org/10.14198//hse.2020.15.Proc3.42

Corresponding author. Department of Physical Therapy for Surgery, Faculty of Physical Therapy, Cairo University, Giza, Egypt. E-mail: m.mana.qeshm@gmail.com

Supplementary Issue: Spring Conferences of Sports Science. International Conference on Psychology of Education Sciences and Lifestyle.

JOURNAL OF HUMAN SPORT \& EXERCISE ISSN 1988-5202

(c) Faculty of Education. University of Alicante

doi:10.14198/jhse.2020.15.Proc3.42
\end{abstract}




\section{INTRODUCTION}

Epidemiological, clinical and basic research clearly demonstrated that regular exercise could prevent a spread of chronic diseases, like cardiovascular diseases, diabetes, obesity, and enhance overall physical and psychological health from a young age. For example, physically, regular exercise in childhood improves strength and endurance, helps build healthy bones and muscles, helps control weight, improves vital sign, reduces pulse, and increases cholesterol levels (Yetter, 2009). Psychologically, regular exercise can reduce anxiety and stress as well as improve happiness, selfconfidence, and boost self-esteem. The numerous health benefits gained from regular exercise are hooked into the sort, intensity and volume of activity pursued by the individual (Netz, 2005). So, the aim of this study was to see the effect of moderate intensity aerobics training (60\% MHR) read mill training on IBS symptom, associated pain and quality of Life in post cholecystectomy patients. There are no specific laboratory tests or medical imaging tests that can be used to diagnose IBS. Control of irritable bowel syndrome. Parasitic infections, lactose intolerance, overgrowth of bacteria in the small intestine, and celiac disease are recommended for all patients before diagnosis of IBS. It is recommended that patients with the disease over the age of 50 be screened for colonitis.

\section{Symptoms of Irritable Bowel Syndrome}

Early symptoms of irritable bowel syndrome include abdominal pain or discomfort with frequent diarrhoea and constipation, as well as changes in bowel habits. You may also feel the need to defecate, feel incomplete or forceful, bloating, and bloating. People with AIDS are more likely to develop gastroesophageal reflux disease, genital-urinary tract symptoms, psychological symptoms such as depression and anxiety, fibromyalgia, chronic fatigue syndrome, headaches and low back pain.

\section{Prevention of Irritable Bowel Syndrome}

Minimize fried foods, meat, oil, margarine, dairy, and other fatty foods. They can cause the colon to function so badly that it can lead to diarrhoea and abdominal pain. Avoid spicy foods. For example, capsaicin in hot peppers causes the colon to spasm, which can lead to diarrhoea.

Reduce caffeine consumption. It can cause IBS because it irritates the large intestine. Avoid bloating foods, including cabbage, Brussels sprouts, broccoli. Do not use chewing gum or candies containing artificial sugars. Among the common sweeteners, sorbitol and mannitol have a laxative effect, but are very difficult to digest. When the gut bacteria try to break down these "non-absorbable sugars", they produce gas and lead to diarrhoea. Stop smoking. Nicotine helps with IBS. Also, when you smoke, you inhale air, and people with IBS are very sensitive to having air in their gut.

\section{SUBJECTS, MATERIALS AND METHODS}

\section{Subjects}

So, Thirty post cholecystectomy patients, both sexes, complaining from Irritable Bowel Syndrome were recruited for this study, their age ranged from 25 to 40 years. Patients were selected from Kasr Al Ainy Hospital in the period between Oct 2017 and Feb 2019. The inclusive criteria were medically stable patients who had a cholecystectomy operation 3 weeks ago. patients were excluded if they had cardiovascular or orthopaedic problems, neurological or musculoskeletal disorders, mental disorder (it makes cooperation impossible),renal failure, myocardial infarction, severe asthma, diabetes, hypertensive, autoimmune disease or smokers subjects. 


\section{Sampling and randomization}

Randomization was used to eliminate the researches' bias. Group A: This group included 15 post cholecystectomy patients complaining from irritable bowel syndrome. They participated in 4 weeks of moderate intensity aerobic educational program (60\% MHR Treadmill Training) (3sessions per week) plus receiving medical treatment protocol.

Group B: This group included 15 non-athletic post cholecystectomy patients complaining from irritable bowel syndrome. They received the routine medical treatment protocol only. Medical treatment were Dietary treatment, psychological treatment, and pharmacological treatments outlines for IBS (Antidepressants, Fibres and laxatives for IBS-C, Antidiarrheal agents for IBS-D and Serotonin receptor agonists/antagonists) (Spiller et al., 2007). Figure (1).

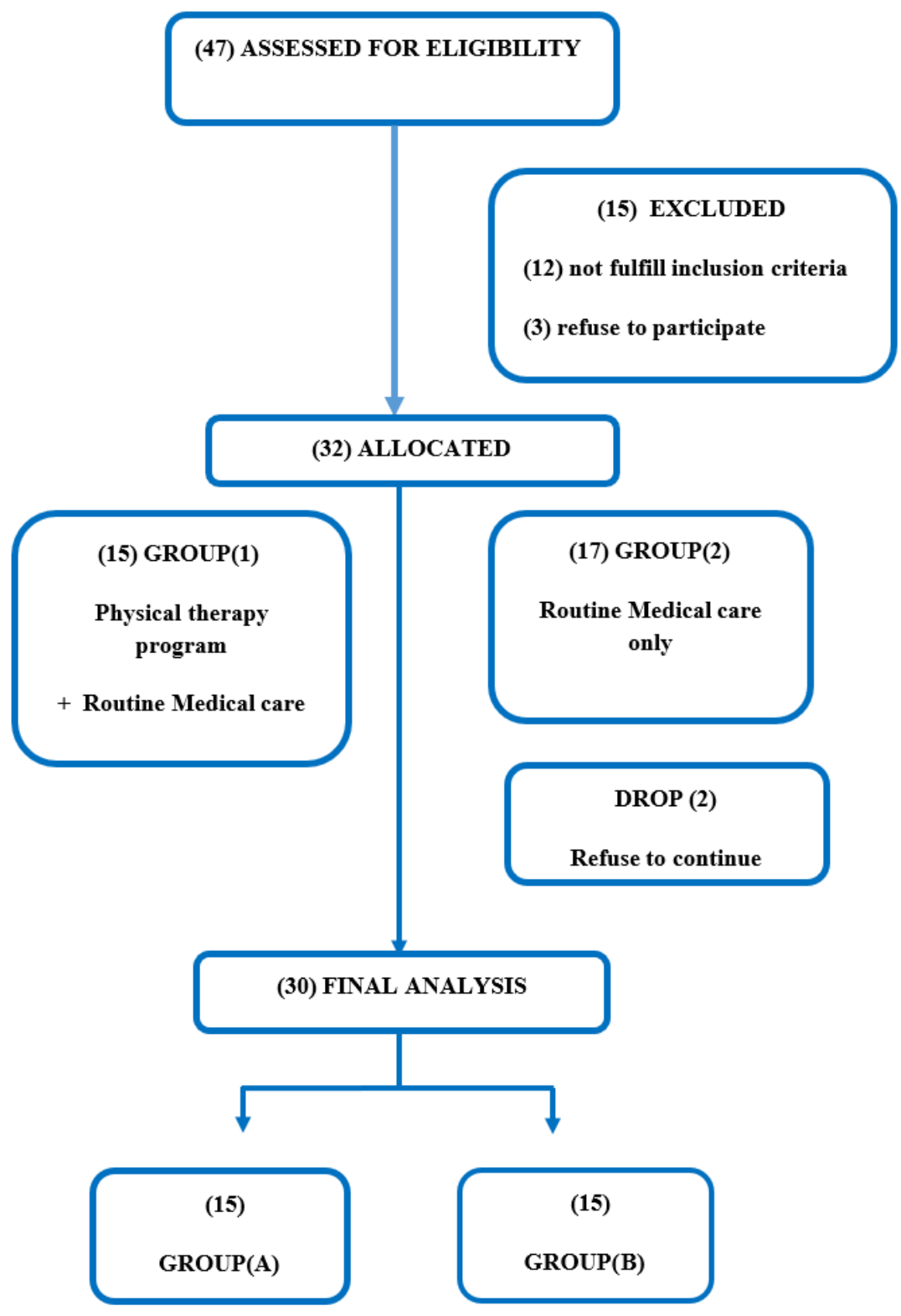

Figure 1. Schematic of the research methodology. 


\section{Instrumentations}

\section{Evaluating equipment and methods}

Standard weight scale: to measure weight and height and body mass index (BMI) then calculated for every patient.

Pulse oximeter: equipment was used to measure the heart rate during exercise and to determine the training heart rate and insurers that the patient reach the desired heart rate during exercise.

Irritable Bowel Syndrome (IBS) Severity Score Procedures: The questionnaire has pain question, abdominal distension question, satisfaction with bowel habit and the way much the IBS been affecting or interfering with patients life generally, The responses to all questions was measured on a ten points scale from (0) no distension to (10) very severe (Spiller et al., 2007\& Drossman et al.,2011).

Irritable Bowel Syndrome Quality of Life (IBS-QOL) Instrument: The questionnaire consists of 32 questions asks about the effect of IBS and therefore the psychological, social and daily living activates and therefore the scale are often subdivided into 7 subscale namely Dysphoria section, Interference with activity, body image, health worry, food avoidance, social reaction, sexual, and relationship (Drossman et al., 2000 \& Andrae et al., 2013).

\section{Treatment equipment}

Treadmill JOHNSON T6000 AC MOTOR was used for training. The maximum speed of treadmill is 25 $\mathrm{km} / \mathrm{h}$. the treadmill can be inclined and lifting $150 \mathrm{~kg}$.

\section{Treatment procedures}

Both groups ( $A$ and $B$ ) received an equivalent medical treatment program throughout the treatment period. additionally, to the medical treatment, Group $(A)$ received moderate intensity aerobic exercises program of exercise with intensity $60 \%$ MHR and frequency three times/week for 4 weeks.

\section{Statistical analysis}

Data were collected before initiation of the treatment and 4 weeks after treatment. Collected data were statistically analysed by descriptive statistics, age, height, weight, BMI, IBS-SSS and IBS-QOL variables were calculated for all patients. Inferential statistical analysis and comparison of IBS-SSS and IBS-QOL were made by paired sample t-tests for within group comparison, between pre and post treatment results, while independent sample t-test was used for comparison between groups at pre and post-treatment periods. Independent t-test was used for comparison between groups age (years), weight $(\mathrm{Kg})$, height $(\mathrm{cm})$ and $\mathrm{BMI}(\mathrm{kg} / \mathrm{m} 2)$ variables. the extent of significance was set at the .05 level.

\section{RESULTS}

Data for the body mass, height, BMI, IBS-SSS or IBS-QOL are presented in Table 1.

\section{IBS-SSS results}

Between groups comparison for mean values of IBS-SSS showed significant difference post treatment with $(p<.0001)$ between both groups in group $A$ than group $B$. 
Table 1. General characteristics of the two groups (A and B groups).

\begin{tabular}{lccccc}
\hline \multirow{2}{*}{ Items } & Group A & Group B & \multicolumn{3}{c}{ Comparison } \\
\cline { 2 - 6 } Age $(\mathrm{yrs})$. & Mean \pm SD & Mean \pm SD & t-value & p-value & S \\
\cline { 2 - 6 } Weight $(\mathrm{Kg})$ & $31.6 \pm 4.579$ & $32.13 \pm 5.29$ & -0.295 & .77 & $\mathrm{NS}$ \\
Height $(\mathrm{cm})$ & $71.87 \pm 11.426$ & $77.6 \pm 8.959$ & -0.441 & .662 & $\mathrm{NS}$ \\
BMl $(\mathrm{Kg} / \mathrm{m} 2)$ & $169.67 \pm 9.854$ & $171.13 \pm 8.28$ & -1.529 & .137 & $\mathrm{NS}$ \\
IBS-SSS & $24.93 \pm 3.173$ & $26.47 \pm 2.232$ & -1.531 & .137 & $\mathrm{NS}$ \\
IBS-QOL & $382.667 \pm 31.045$ & $376.667 \pm 33.947$ & 0.505 & .617 & $\mathrm{NS}$ \\
& $139.533 \pm 11.025$ & $144.8 \pm 7.523$ & -1.528 & .138 & $\mathrm{NS}$ \\
\hline
\end{tabular}

Table 2. Comparison between IBS-SSS results of Group A \& Group B before \& after treatment.

\begin{tabular}{lllll}
\hline \multirow{2}{*}{ Item } & \multicolumn{2}{c}{ Before treatment } & \multicolumn{2}{c}{ After treatment } \\
\cline { 2 - 5 } Mean & Group A & Group B & Group A & Group B \\
\cline { 2 - 5 } \pm SD & 382.667 & 376.667 & 112.000 & 182.000 \\
Mean difference & 31.045 & 33.947 & 13.732 & 17.809 \\
t-value & & 6.000 & \multicolumn{2}{c}{-70.000} \\
p-value & \multicolumn{2}{c}{-12.056} & \multicolumn{2}{c}{.000} \\
\hline
\end{tabular}

\section{IBS-QOL results}

Regarding IBS-QOL Table 3 showed significant reduction of IBS-QOL score at post treatment in comparison to pre-treatment in both groups $A$ and $B$ with $p$-value $=.138$. Between groups comparison of IBS-QOL showed significant difference post treatment with $(p<.0001)$ between both groups in favour to group $A$.

Table 3. Comparison between IBS-QOL results before \& after treatment in Group A \& Group B.

\begin{tabular}{lcccc}
\hline \multirow{2}{*}{ Item } & \multicolumn{2}{c}{ Before treatment } & \multicolumn{2}{c}{ After treatment } \\
\cline { 2 - 5 } Mean & Group A & Group B & Group A & Group B \\
\cline { 2 - 5 } \pm SD & 139.533 & 144.800 & 60.333 & 94.267 \\
Mean difference & 11.025 & 7.523 & 6.883 & 5.257 \\
t-value & \multicolumn{2}{c}{-5.267} & \multicolumn{2}{c}{-33.933} \\
p-value & \multicolumn{2}{c}{-1.528} & \multicolumn{2}{c}{-15.174} \\
\hline
\end{tabular}

\section{DISCUSSION AND CONCLUSION}

Our study is performed to investigate the impact of moderate intensity aerobic movement on patients with irritable bowel syndrome post cholecystectomy. The pre- treatment results of the present study revealed no significant difference between the mean values of IBS-SSS and IBS-QOL between both groups (Group A Group B). The post - treatment results of this study by IBS-SSS showed that there was Significant difference between both groups in favour to group $A$. the result of this study prove that moderate intensity aerobic exercises plus the routine medical care can play an important role in reducing post cholecystectomy IBS and improving patient quality of life. Yanor-McRae,2000, explained that, psychotherapy, biofeedback, herbs, relaxation training, visualization, and acupuncture may offer more control and relief over symptoms than conventional medication therapies, also regular exercise recommended, because it tones the abdominal muscles and can stimulate digestive organs to be more active and balanced. Regarding the effect of the moderate intensity aerobic exercises on irritable bowel 
syndrome, our results came in consistence with Maleki et al.,2018, Johannesson et al.,2011 ,Morlin et al.,2008, De Schryver et al.,2005, Villoria et al.,2006, Lustyk et al.,2001, Feng et al2010,\&, Dely et al.,2008, through their studies found that aerobic exercises can play an important role in reducing IBS and improving patient life quality. From the results of current study and the results of previous studies, it can be concluded that moderate intensity aerobic exercise training $(60 \% \mathrm{MHR})$ for 4 weeks is effective in treatment of IBS post cholecystectomy through reducing IBS symptoms and improving Quality of Life.

\section{REFERENCES}

Andrae, D.A., (2013); Evaluation of the Irritable Bowel Syndrome Quality of Life (IBS-QOL) questionnaire in diarrheal-predominant irritable bowel syndrome patients. Health and quality of life outcomes,. 11(1): p. 208. https://doi.org/10.1186/1477-7525-11-208

Blanchard, E.B. and Scharff, L.( 2002); Psychosocial aspects of assessment and treatment of irritable bowel syndrome in adults and recurrent abdominal pain in children. Journal of Consulting and Clinical Psychology. 70(3), 725-738. https://doi.org/10.1037/0022-006X.70.3.725

Blanchard, E.B., L.J.J.o.c. Scharff. (2002); Psychology, psychosocial aspects of assessment and treatment of irritable bowel syndrome in adults and recurrent abdominal pain in children,. $70(3)$ : $p$. 725. https://doi.org/10.1037/0022-006X.70.3.725

Canavan, C., J. West, and T.R. Card (2014); The epidemiology of irritable bowel syndrome. Clinical epidemiology, 6: p. 71-80. https://doi.org/10.2147/CLEP.S40245

Chey, W. D., and Rai, J. (2011); Exercise and IBS: No Pain, No Gain. Gastroenterology, 141(5), 1941 1943. https://doi.org/10.1053/i.gastro.2011.09.030

Daley, A., (2008); The effects of exercise upon symptoms and quality of life in patients diagnosed with irritable bowel syndrome: A randomized controlled trial. International journal of sports medicine, 29(09): p. 778-782. https://doi.org/10.1055/s-2008-1038600

Drossman, D.A., (2011) ; Severity in irritable bowel syndrome: A Rome foundation working Team report. The American journal of gastroenterology,. 106(10): p. 1749. https://doi.org/10.1038/ajg.2011.201

Feng, Y., ( 2010); Observation of the efficacy of Baduanjin exercise on the constipation-predominant irritable bowel syndrome of the elderly. Sport Sci Res,. 31(2): p. 89-98.

Hill, E.,( 2008); Exercise and circulating cortisol levels: The intensity threshold effect. Journal of endocrinological investigation, 31(7): p. 587-591. https://doi.org/10.1007/BF03345606

Jaunoo, S., S. Mohandas, and L. Almond,( 2010); Postcholecystectomy syndrome (PCS). International journal of surgery, 8(1): p. 15-17. https://doi.org/10.1016/i.jisu.2009.10.008

Johannesson, E., Ringström, G., Abrahamsson, H., and Sadik, R.( 2015); Intervention to increase physical activity in irritable bowel syndrome shows long-term positive effects. World journal of gastroenterology: WJG, 21(2), 600. https://doi.org/10.3748/wig.v21.i2.600

Johannesson, E., Simrén, M., Strid, H. (2011); Physical activity improves symptoms in irritable bowel syndrome: A randomized controlled trial. The American journal of gastroenterology, 106(5), 915-922. https://doi.org/10.1038/ajg.2010.480

Keteyian, S.J.,(2001); Graded exercise testing and exercise prescription., In clinical exercise physiology, J.K. Ehrman, Editors. 2013, Human Kinetics: Champaign, Illinoi. p. 61-88.

Levy, R.L., (2001); Irritable bowel syndrome in twins: Heredity and social learning both contribute to etiology. Gastroenterology, 121(4): p. 799-804. https://doi.org/10.1053/gast.2001.27995

Lustyk, M., (2001); Does a physically active lifestyle improve symptoms in women with irritable bowel syndrome? Gastroenterology nursing: the official journal of the Society of Gastroenterology Nurses and Associates, 24(3): p. 129-137. https://doi.org/10.1097/00001610-200105000-00007 
Maleki, B. H., Tartibian, B., Mooren, F. C., FitzGerald, L. Z., Krüger, K., et al.( 2018); Low-to-moderate intensity aerobic exercise training modulates irritable bowel syndrome through antioxidative and inflammatory mechanisms in women: Results of a randomized controlled trial. Cytokine, 102, 18-25. https://doi.org/10.1016/j.cyto.2017.12.016

Netz, Y., (2005); Physical activity and psychological well-being in advanced age: A meta-analysis of intervention studies. Psychology and aging, 20(2): p. 272. https://doi.org/10.1037/08827974.20 .2 .272

Spiller, R., (2007); Guidelines on the irritable bowel syndrome: Mechanisms and practical management. J Gut, 56(12): p. 1770-1798. https://doi.org/10.1136/gut.2007.119446

Yanor-McRae. R.(2000) ; Calm your irritable bowel. Canadian Journal of Health and Nutrition,. 218, 3638.

Yetter, G.,(2009); Exercise-based school obesity prevention programs: An overview. Psychology in the Schools, 46(8): p. 739-747. https://doi.org/10.1002/pits.20412

\section{(c) 7 () $\Theta$}

This work is licensed under a Attribution-NonCommercial-NoDerivatives 4.0 International (CC BY-NC-ND 4.0). 